\title{
MicroRNas: beating cancer with new powerful weapons
}

\author{
Diana Gulei ${ }^{1}$, Ioana Berindan-Neagoe ${ }^{1,2,3}$, George A Calin ${ }^{4,5 *}$ \\ ${ }^{1}$ MEDFUTURE - Research Center for Advanced Medicine, \\ "Iuliu Hatieganu" University of Medicine and Pharmacy, Cluj-Napoca, Romania \\ ${ }^{2}$ Research Center for Functional Genomics, Biomedicine and Translational Medicine, \\ "Iuliu Hatieganu" University of Medicine and Pharmacy, Cluj-Napoca, Romania \\ ${ }^{3}$ Department of Functional Genomics and Experimental Pathology, \\ The Oncological Institute "Prof. Dr. Ion Chiricuta", Cluj-Napoca, Romania \\ ${ }^{4}$ Department of Experimental Therapeutics, The University of Texas MD Anderson Cancer Center, Houston, Texas, USA \\ ${ }^{5}$ Center for RNA Interference and Non-coding RNAs, The University of Texas MD Anderson Cancer Center, \\ Houston, Texas, USA
}

(Received 8 April, 2017; accepted 8 May, 2017)

\begin{abstract}
MicroRNAs have become central players in the process of finding and implementing novel therapeutic strategies for the overcoming of the cancer associated heterogeneous effects. Their ability to target multiple coding genes in order to influence and regulate entire signaling networks and also their differential expression between healthy and malignant cell is nowadays tackled in preclinical studies that enroll models of human cancers. The main approaches consist in the inhibition of oncogenic miRNAs that are usually overexpressed in transformed cells and also the re-expression of the tumor suppressor ones that are characterized by a downregulated expression pattern. No matter the strategy, it is vital to choose wisely the targeted sequence in order to achieve global cancer inhibitory effects, where key players that direct phenotypically transformations sustain numerous miRNA signatures. As important is also the therapeutic strategy that needs to be validated in terms of both efficiency and safety in order to avoid installation of secondary pathological conditions.
\end{abstract}

Keywords: miRNA, therapy, cancer, inhibitors, mimics, delivery

\section{INTRODUCTION}

MicroRNAs (miRNAs) are small, non-coding sequences of approximately 19-25 nucleotides that are involved in the regulation of diverse target genes through direct binding to the $3^{\prime}$ untranslated region (UTR) of the targeted messenger RNA (mRNA) transcripts (more rarely the binding can take place also in the 5 '-end or coding sequence). The complementarity between the two transcripts (miRNA and mRNA) allows the sequence specific hybridization that concludes with the inhibition of translational expression of the target gene or, more rarely, even with the degradation of the mRNA. The binding of miRNAs to the target gene does not require perfect complementarity and in this way it is allowed a multiple target allocation, where the same sequence can inhibit the expression of more than one gene and the same gene can be targeted by multiple miRNAs. In this way it is created a complex regulatory network that sustains and control key processes within the organism (1-3).

In the same time, multiple diseases are associated with pathological expression of these miRNAs, including cancers that present different aberrant non-coding signatures. These signatures are often cancer type specific, and moreover can even differentiate between cancer subtypes or progression stages or associate with the patients' survival $(1,4,5)$.

Despite their emerging role as diagnosis and prognosis tools, miRNAs have become the central players for the development of a new type of cancer management. MiRNA therapies are nowadays explored in a variety of preclinical studies performed across the world in order to introduce this strategy in clinical scenarios. Their small size represent an attractive characteristic for the development of targeted therapies, based on suppression of the expression of some oncogenic miRNAs (miRNA

*Corresponding author (Email: gcalin@mdanderson.org)) 
inhibition therapy) or reinforced the levels of the tumor suppressor ones (miRNA replacement therapy) $(2,6)$.

This review will briefly discuss the most recent developments on the subject of miRNA therapeutics in cancer together with some directions towards the safe and efficient administration in humans.

\section{MicroRNAs - FROM BIOGENESIS TO GENE REGULATION}

The biogenesis of miRNAs starts once the RNA Polymerase II (or in certain cases RNA Polymerase III) begins to transcribe intragenic or intergenic sequences compromising miRNAs loci. The resulted transcript (primary RNA, pri-miRNA) is significantly longer than the final product and presents itself as a stem-loop structure that is $5^{\prime}$ capped and $3^{\prime}$ polyadenylated. Enzymatic processing steps mediated by Drosha in the nucleus and Dicer in the cytoplasm accompany their exportation in the cytoplasm and subsequent maturation. Once the resulted miRNA/miRNA* duplex is divided into two single stranded sequences (the miRNA and the passenger miRNA strand, or miRNA*, see Fig. 1), the mature active miRNA (miRNA) is taken by RISC (RNA-Induced Silencing Complex) and under this form it can exert regulatory roles regarding the levels of gene expression through complementary binding with the target transcripts (7-9). Following miRNA-target interaction the translational process is impairing by "seed" (miRNA regions of 6-8 nucleotides that mediate the hybridization process) complementarity and gene expression is inhibited (7).

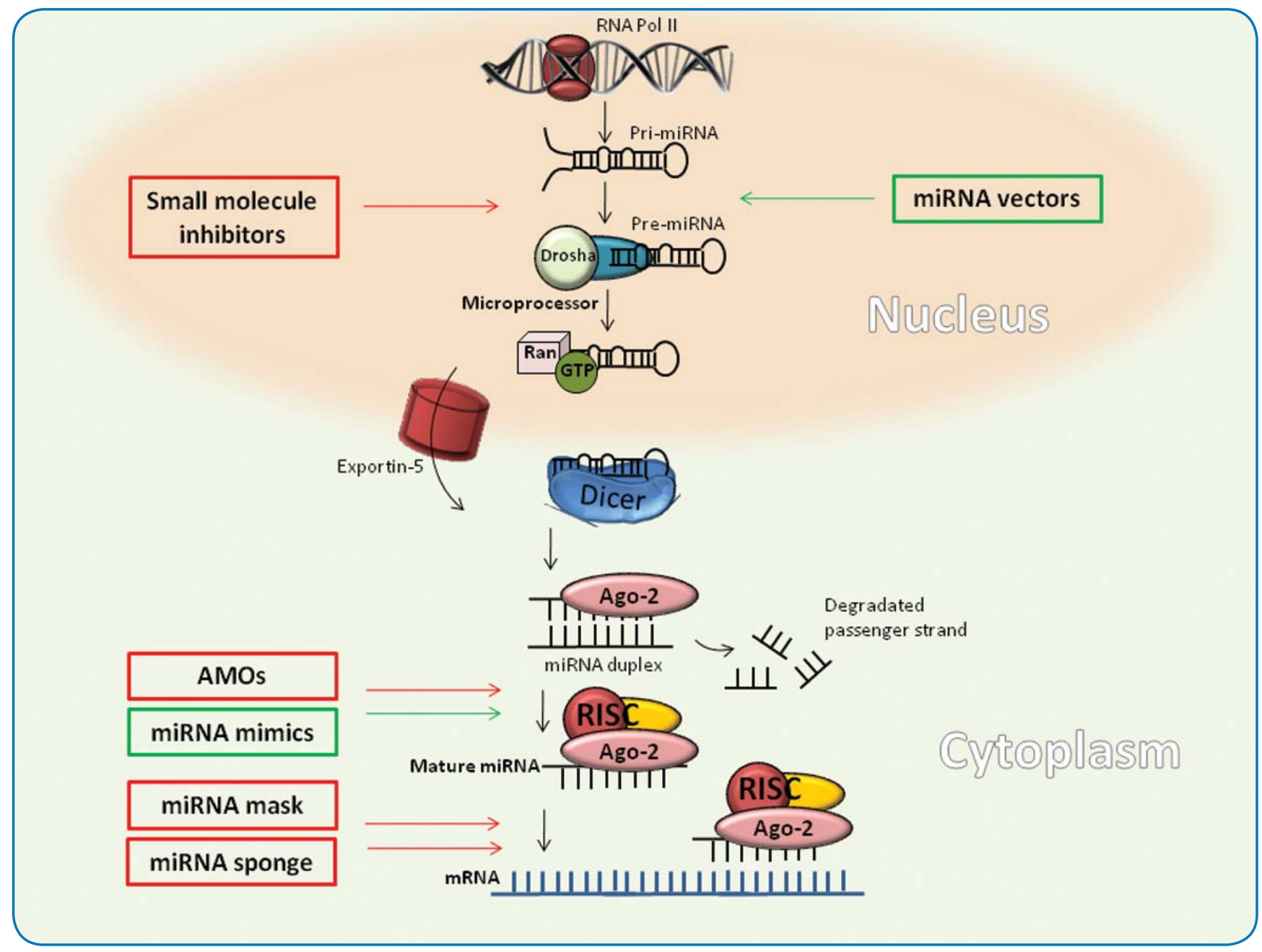

Fig. 1. - Therapeutic alternatives in targeting miRNA regulation. The biogenesis of miRNAs and the interaction with the target gene involves a series of steps that can be targeted for miRNA modulation: RNA Pol II transcribes the sequences associated with miRNA loci, resulting in a stem-loop pri-miRNA 5' capped and 3' polyadenylated; Drosha removes the overhangs, where pre-miRNA is then taken by Ran-GTP in order to be exported in the cytoplasm via Exportin-5; Dicer continues the enzymatic processing steps, creating a miRNA duplex, where the guide strand associates with Ago-2 and the passenger one is degraded; the mature miRNA associates with RISC and under this form is able to target and regulate the expression of the transcript (mRNA). Green box - miRNA replacement strategy; Red box - miRNA inhibition strategy Abbreviations: $\mathrm{miRNA}=$ microRNA; RNA Pol II=RNA Polymerase II; Ran=RAs-related Nuclear protein; GTP=Guanosine-5'-triphosphate; Ago-2=Argonaute-2; RISC=RNA-induced silencing complex; mRNA=messenger RNA; AMOs=Anti-miRNA Oligonucleotides 
Even if the consecrated concept explaining miRNAs mediated activity is represented by translational repression through miRNA-mRNA interaction, miRNAs are also able to stimulate gene expression. It was shown that let-7, a well-studied miRNA, is capable to induce up-regulation of the target transcript in cases of cell cycle arrest, although the same non-coding transcript is hampering the translation in proliferating cells (10). The regulatory interactions can also take place at protein levels, where miRNAs exhibit decoy activity by binding directly to target proteins, as in the case of miR-328 in leukemic blasts (11). More recently, it was discovered that miRNAs are also involved in signaling mechanism through direct binding with several receptors, acting as ligands in order to stimulate downstream pathways. As an example, Toll-like receptor 8 can be activated by miR-21/miR-29a discharged by malignant cells via exosomes, concluding with the installation of an inflammatory microenvironment upon cytokine release (e.g., TNF- $\alpha$ and IL-6), that in the end could promote cancer development and spreading (12).

All together, these regulatory schemes contour a complex network where miRNAs play extensive roles within the organism in both normal and pathological processes.

\section{THE MAIN miRNAs INVOLVED IN CANCER}

Differential expression of miRNAs between healthy and malignant cells is driven by multiple factors and is also one of the essential features that allow cancer development. A simplistic approach in describing the malignant signatures revolving around miRNAs is referring to the main stratification of the non-coding molecules in tumor suppressors and oncogenic ones that target inversely correlated coding genes. Usually, the tumor suppressors are downregulated and the oncogenic miRNAs are upregulated in comparison with the profiles of healthy cells (13). Transgenic mice models overexpressing oncogenic miR-21 or miR-155 are developing acute B cell leukemia $(14,15)$, while knockout mice for miR-15a/16-1 cluster acquire chronic lymphocytic leukemia due to elimination of the tumor suppressor features of the cluster (16). Moreover, the same miRNA can sustain the malignant progression in one type of pathology and suppress the tumor spreading in others due to alternative target genes.

The regulatory role of the non-coding transcripts is extending upon all cancer hallmarks implemented by Hanahan and Weinberg in 2000 and updated in 2011 $(17,18)$.

Let-7 family is the first described miRNA family, hence the different annotation within the small non-coding group (19). These transcripts are generally associated with tumor suppressor functions, where the corresponding genes are commonly altered or are even missing in numerous malignant pathologies (20). Among the main oncogenes targeted by let- 7 members are Ras and
HMGA2 (high mobility group AT-hook 2) (let-7 group has numerous "seed" identical members, beginning with let-7a and ending with let-7i and miR-98, able to target the same gene). Ras is identified as mutated in a heterogenic panel of cancers, being involved as a GTPase transducer in cell survival, proliferation and growth (21). HMG2A is a transcription factor involved in similar process as Ras (22) and is detected in high levels especially in lung cancer (23). Loss of let-7 family member expression is often characterized by increased levels of these oncogenes concomitant with promotion of cell growth and proliferation, enforcing the possible enrolment of let-7 into miRNA therapy.

MiR-155 is one of the most studied microRNA, with functional activities that spread beyond simplistic regulatory signaling pathways, being involved in numerous interconnected networks within malignant pathologies, infections, nervous system disorders, immune system associated conditions, cardiac diseases and others. Therefore, miR-155 ranks among the most relevant molecules for therapeutic strategies $(24,25)$. In terms of cancer abnormalities, the specific miR-155 sequence holds a pertinent role both in hematological and solid malignancies, being classified generally as an oncogenic miRNA, with some limited exceptions known till now where it acts as a suppressor (26).

\section{MiRNA THERAPEUTICS IN CANCER}

The differential expression of miRNAs between healthy and cancer cells has conducted to the establishment of different miRNA signatures containing several key non-coding sequences proposed nowadays as therapeutic targets. In this sense, therapeutic strategies based on miRNA modulation are expected to pass into the clinic in the next few years in order to enhance the management of cancer patients (27-29).

One of the main provocations in terms of cancer therapy, and not only, is represented by the selection of the right target, a target that once modulated will be able to significantly alter the malignant phenotype. This issue is also present in the case of miRNA therapy, where regardless of their important modulatory role only few sequences are actually able to orchestrate extensive carcinogenic signaling pathways. Therefore, researchers are now concentrating on a quite restrictive number of miRNAs that have been shown to play key roles within cancer development and spreading in order to slowly pave their way into the clinic (30).

One such example is miR-34a, a member of the miR-34 family, that is engaged in targeting of approximately 30 oncogenes and several other genes involved in immune evasion of cancer cells. This sequence is found as downregulated in multiple types of cancers, being repetitively called across the scientific literature as a master 
tumor suppressor (30). As a result of this extensive network coordinated by miR-34 that is further lost in malignant scenarios, this was the first miRNA tested in human patients with primary liver cancer and other selected solid or hematological malignancies (MRX34 - clinical trial information: NCT01829971). The therapeutic scheme consisted in intravenously administration of a liposomal miR-34a mimic for five consecutive days, followed by a two weeks pause, cycle repeated by 3 times. Unfortunately, on 20 September 2016, the company Mirna Therapeutics, Inc. (Nasdaq:MIRN) announced the closure of the Phase I clinical trial due to multiple immune-related severe adverse events (SAE) in five tested patients. This situation highlights the importance of preclinical toxicology studies of miRNA related therapeutics, despite the promising tumor inhibition effects.

MiR-155 is generally associated with oncogenic functions, being often upregulated in different cancers, especially hematological malignancies and lung and breast cancer. The general trend consists in inhibition of miR-155 that demonstrated significant effects on transformed cells with inhibition of cellular growth and proliferation, activation of apoptosis and weakening of metastatic spread. All these effects represent the consequence of impairing miR-155 ability to interact from both structural and functional points of view with key genes within the malignant installation and development (31-34). A recent study performed by Van Roosbroecket et al. revealed a negative feedback between miR-155 and TP53 (an essential tumor suppressor gene) together with a role of this interplay in the installation of multidrug resistance. Also, in vivo modulation of miRNA with anti-miR155 using an orthotopic lung cancer model showed decreased chemoresistance together with inhibition of cancer parameters. Moreover, they extended their study and tested the effects of anti-miR155 in combination with chemotherapeutic drugs, a superior approach that considerably limits the amount of necessary administrated therapeutic components, and therefore reduce the potential of adverse reactions. Not less important, they also demonstrated through in vivo toxicology studies that anti-miR-155-DOPC can be considered safe for administration in the clinic, since no adverse effects were shown (31).

There are also some other miRNAs or miRNA combinations proposed for potential testing in humans based on the promising results obtained in vitro and in vivo. Let-7 together with miR-34 mimics inhibited tumor growth in an in vivo model of non-small cell lung cancer (NSCLC) by increasing the level of oncogenes inhibition like Myc, Lin28b and c-Met (35). Also concentrated on lung cancer, another group tested the hypothesis of miR-200c replacement and revealed positive results regarding the sensitivity of tumor cells to radiation following the subsequent inhibition of RAD51, SESN1 and Nrf2 (36). The effects of miR-21 knockdown via lentiviral vectors (LVs) were tested in pancreatic cancer, alone or in combination with gemcitabine. This strategy was associated with tumor regression and it was proposed also for other types of cancer, considering the extensive involvement of miR-21 in numerous malignant diseases (37). Mir-25 overexpression in colon cancer was also shown to be effective in vitro and in vivo most probably due to restored direct inhibition Smad-7 (38).

These examples as well as many others sustain the importance of miRNAs in cancer progression and also their potent role as therapeutics. However, these preclinical studies need to take in consideration numerous parameters especially toxicity and immune related adverse effects, in order to be further validated in humans. Considering the fact that most of the studies are made on immunocompromised mice that can bear human tumor grafts, it is hard to exactly predict the changes that will appear as a response to miRNA inhibition or replacement. We need to tackle this problem through enrolment of humanized mice or immuno-avatars that are able to offer information regarding the modifications in the immune panel, raising the level of reliability in terms of clinical simulation.

\section{MiRNAs DELIVERY}

The establishment of the crucial role of miRNAs in the initiation and progression of malignant pathologies has conducted towards development of therapeutic strategies that involved the manipulation of miRNA levels within the cell through administration of inhibitory or mimetic molecules (Table 1). In this sense, there are currently two main approaches by which researchers are trying to restore the normal levels of a specific non-coding sequence: inhibition and replacement strategies. In terms of miRNA silencing, there is the possibility to deliver anti-miR oligonucleotides (AMOs), miRNA sponges, antagomiRs and also miRNA masks, where in the case of miRNA replacement, the main approaches consist in the elevation of miRNA levels through "mimic" sequences (2).

\section{MiRNA inhibition strategies}

This type of strategy refers at oncogenic miRNAs that are highly expressed in tumor cells and are targeted for inhibition with complementary sequences that impair the processing of endogenous miRNA by RISC. There were also attempts of targeting other earlier steps in the biogenesis pathway: the loop structure of the pre-miRNA sequence (39), inhibition of the enzymatic processing steps by Drosha or Dicer with morpholino AMOs targeted towards pre-miRNA and pri-miRNA sequences (40). These strategies have limited applications due to the slow turnover of mature miRNAs in the mammalian cells, which shortens the window for inhibition 
Table 1. MiRNA delivery strategies

\begin{tabular}{|c|c|c|c|c|c|}
\hline Therapeutic strategy & Agent & Advantages & Limitations & $\begin{array}{c}\text { Therapeutic } \\
\text { status }\end{array}$ & Ref. \\
\hline \multirow{4}{*}{$\begin{array}{c}\text { Inhibition of } \\
\text { oncogenic miRNAs }\end{array}$} & $\begin{array}{l}\text { Chemically } \\
\text { modified AMOs }\end{array}$ & $\begin{array}{l}\text { Stable, } \\
\text { biodistribution, } \\
\text { bioavailability }\end{array}$ & $\begin{array}{l}\text { High doses in order to obtain a } \\
\text { good inhibition; } \\
\text { possible off-target } \\
\text { effects; toxicity }\end{array}$ & $\begin{array}{l}\text { Preclinical } \\
\text { studies }\end{array}$ & $(2,53)$ \\
\hline & $\begin{array}{l}\text { LNA } \\
\text { anti-miRs }\end{array}$ & $\begin{array}{l}\text { Biodistribution, } \\
\text { effective }\end{array}$ & Possible off-target effects & $\begin{array}{l}\begin{array}{l}\text { Phase I and 2a } \\
\text { (for HCV) }\end{array} \\
\end{array}$ & $\begin{array}{l}(54, \\
55)\end{array}$ \\
\hline & miRNA sponge & $\begin{array}{l}\text { Possibility to silence } \\
\text { entire miRNA } \\
\text { families }\end{array}$ & $\begin{array}{l}\text { Low stability; Delivery; } \\
\text { Possible off-target effects }\end{array}$ & $\begin{array}{l}\text { Preclinical } \\
\text { studies }\end{array}$ & $(2,53)$ \\
\hline & AntagomiRs & $\begin{array}{l}\text { Highly stable, } \\
\text { biodistribution, } \\
\text { bioavailability, } \\
\text { high persistence }\end{array}$ & $\begin{array}{l}\text { High doses to obtain a good } \\
\text { inhibition; possible off-target } \\
\text { effects; toxicity }\end{array}$ & $\begin{array}{l}\text { Preclinical } \\
\text { studies }\end{array}$ & (2) \\
\hline \multirow[t]{2}{*}{ miRNA replacement } & miRNA mimics & $\begin{array}{l}\text { High stability, } \\
\text { highly effective; } \\
\text { nanotechnology } \\
\text { based conjugation }\end{array}$ & $\begin{array}{l}\text { Possible off-target effects; } \\
\text { toxicity }\end{array}$ & Phase I & $(2,53)$ \\
\hline & $\begin{array}{l}\text { miRNA } \\
\text { expression } \\
\text { vectors }\end{array}$ & $\begin{array}{l}\text { Long term } \\
\text { expression; efficient } \\
\text { transduction; } \\
\text { possibility to replace } \\
\text { multiple miRNAs }\end{array}$ & $\begin{array}{l}\text { Possible off-target effects; } \\
\text { potential dose dependent } \\
\text { toxicity }\end{array}$ & $\begin{array}{l}\text { Preclinical } \\
\text { studies }\end{array}$ & $(2,53)$ \\
\hline
\end{tabular}

(41). In these sense, the main strategy remains the targeting of mature miRNA through administration of anti-miR oligonucleotides (AMOs), miRNA sponges, antagomiRs and miRNA masks (2).

Anti-miR oligonucleotides (AMOs) are small antisense sequences of approximately 20 oligonucleotides and are considered to be effective in terms of miRNA therapeutics. Naive AMOs that do not bear any modifications are usually subjected to nuclease degradation or have decreased thermodynamic stability. Therefore, AMOs have been modified in different manners in order to achieve a more effective inhibition, although the standard modification remains the addition of methylated hydroxyl groups (2'-OMe) to the 2'-hydroxyl from the C2 (42). To these modified sequences, researchers added different functional groups in order to further increase the efficiency: conjugation with a phosphorothioate (43); incorporation of fluorine (2'-F) or methoxyethyl (2'-MOE) (44). One of the most successful inhibitory attempts was obtained with locked nucleic acid (LNA)-modified AMOs that are designed to link the 2'-O-oxygen to the 4'-position via a methylene linker (45).

MiRNA sponges were introduced in 2007 (46) and can be generally described as mRNA-like sequences that contain multiple binding sites for endogenous miRNA. After transient transfection into the cells, miRNA sponges have proven to be similarly effective as LNA-modified AMOs and, moreover, were able to inhibit more than one member of a miRNA family (46). Following the work of the initial researchers, another group succeeded to stably preserve the expression of miRNA sponges into the cells and also inhibit two different miRNAs at once by incorporating different binding sites (47).

AntagomiRs work on the same principle as AMOs but they are modified through conjugation with cholesterol in order to increase their stability within the organism upon targeted administration. The intravenously injection in mice models of this chemically modified sequences revealed a persistence of approximately 23 days and also a heterogeneous panel of expression sites (43).

Finally, miRNA masks represent a different approach that is not following the pattern of the previously reminded strategies. In this case, the inhibition is gene specific and the target is actually the mRNA that is masked in order to impair the interaction between miRNAs and the transcript. This type of strategy is very effective when the final scope is to preserve the normal expression of a specific gene and not necessarily an entire network or different networks, considering the ability of miRNAs to bind multiple targets (48).

\section{MiRNA replacement strategies}

MiRNA replacement consists in the administration of miRNA mimics in order to restore the normal expression of some downregulated miRNAs that are normally responsible for tumor suppression functions. This type of strategy is more stringent, considering the fact that the 
administrated sequences need to be taken by RISC, the main approaches consisting in the delivery of miRNA mimics or transfection with plasmid or virally encoded miRNA constructs (49).

MiRNA mimics are double stranded sequences that are very similar to the miRNA duplex resulted after Dicer processing with one guide and one passenger strand paired through Watson-Crick binding (50). As in the case of AMOs, miRNA mimics are also modified in similar manners in order to increase their stability within the organism and avoid nuclease degradation: addition of methyl (2'-O-Me), methoxyethyl (2'-MOE), fluorine (2'-F) groups or combinations between them (49). More recently, researchers also tried to engage single stranded miRNA mimics and succeed to obtain miRNA-Argonaute activity but at inferior levels compared to double stranded mimics (51).

An alternative approach consists in the delivery of shRNA or pri-miRNA mimic via plasmids or viral constructs that are superior in terms of stable expression. Another advantage consists in the fact that the same delivery backbone can hold multiple sequences for mature miRNA expression, enabling the possibility of multiplex miRNA targeting (52).

\section{CONCLUSION AND FUTURE PERSPECTIVES}

MiRNAs have gained their role as crucial modulators of both homeostatic and pathological states, with an increased attention regarding malignant pathologies. As we pass through preclinical studies, we need now more than ever to concentrate on the potential side effects of miRNA therapies, since the efficiency is now consecrated in the research community. Even if we are now aware about the immense potential of miRNAs inhibitors or mimics, it is equally important to find a strategy that is safe for human delivery and has limited side effects. One such approach is to combine the current treatments with the novel ones (miRNA therapeutics) in order to diminish the necessary doses and exclude a potential toxicity or even immune reactions. In terms of the immune system it is mandatory to incorporate in the preclinical studies models that are capable to mimic the scenario within a human organism: humanized mice or immuno-avatar. Even if the current xenografts mice represent reliable platforms for different drug testing, they are not able to reveal the complete picture due to depletion of the immune cells. Combining the current knowledge with last generations testing models will improve significantly the management of miRNAs therapeutics and will also permit a more informed transit to the clinic.

Acknowledgements: Work in Dr. Calin's laboratory is supported by National Institutes of Health (NIH/NCATS) grant UH3TR00943-01 through the NIH Common Fund, Office of Strategic Coordination (OSC), the NIH/NCI grant 1 R01 CA182905-01, a U54 grant - UPR/MDACC Partnership for Excellence in Cancer Research 2016 Pilot Project, a CLL Moonshot Flagship project and the Estate of C. G. Johnson, Jr. This work was supported by a POC grant Nr.35/01.09.2016, ID 37_796, entitled "Clinical and economical impact of personalized targeted anti-microRNA therapies in reconverting lung cancer chemoresistance" - CANTEMIR.

The authors have nothing to disclose.

\section{REFERENCES}

1. Berindan-Neagoe I, et al., MicroRNAome genome: a treasure for cancer diagnosis and therapy, CA Cancer J. Clin., 5, 311-336 (2014).

2. Shah MY, et al., microRNA Therapeutics in Cancer-An Emerging Concept, EBioMedicine, 12, 34-42 (2016).

3. Gulyaeva LF, Kushlinskiy NE, Regulatory mechanisms of microRNA expression, J. Transl. Med., 1, 143 (2016).

4. Calin GA, et al., Frequent deletions and down-regulation of micro- RNA genes miR15 and miR16 at $13 q 14$ in chronic lymphocytic leukemia, Proc. Natl. Acad. Sci. U.S.A., 24, 15524-15529 (2002).

5. Braicu C, Calin GA, Berindan-Neagoe I, MicroRNAs and cancer therapy - from bystanders to major players,

Curr. Med. Chem., 29, 3561-3573 (2013).

6. Redis RS, et al., Non-coding RNAs as theranostics in human cancers, J. Cell Biochem., 5, 1451-1459 (2012).

7. Macfarlane LA, Murphy PR, MicroRNA: Biogenesis, Function and Role in Cancer, Curr. Genomics, 7, 537-561 (2010).

8. Bartel DP, MicroRNAs: genomics, biogenesis, mechanism, and function, Cell, 2, 281-297 (2004).

9. Kim VN, MicroRNA biogenesis: coordinated cropping and dicing,

Nat. Rev. Mol. Cell Biol., 5, 376-385 (2005).

10. Vasudevan S, Tong Y, Steitz JA, Switching from repression to activation: microRNAs can up-regulate translation,

Science, 5858, 1931-1934 (2007).

11. Eiring AM, et al., miR-328 functions as an RNA decoy to modulate hnRNP E2 regulation of $m R N A$ translation in leukemic blasts, Cell, 5, 652-665 (2010).

12. Fabbri M, et al., MicroRNAs bind to Toll-like receptors to induce prometastatic inflammatory response, Proc. Natl. Acad. Sci. U.S.A., 31, E2110-E2116 (2012).

13. Garzon R, et al., MicroRNA expression and function in cancer, Trends Mol. Med., 12, 580-587 (2006).

14. Medina PP, Nolde M, Slack FJ, OncomiR addiction in an in vivo model of microRNA-21-induced pre-B-cell lymphoma,

|Nature, 7311, 86-90 (2010).

15. Costinean S, et al., Pre-B cell proliferation and lymphoblastic leukemialhigh-grade lymphoma in E(mu)-miR155 transgenic mice, Proc. Natl. Acad. Sci. U.S.A., 18, 7024-7029 (2010).

16. Klein, U., et al., The DLEU2/miR-15a/16-1 cluster controls B cell proliferation and its deletion leads to chronic lymphocytic leukemia, Cancer Cell, 1, 28-40 (2010). 
17. Hanahan D, Weinberg RA, The hallmarks of cancer, Cell, 1, 57-70 (2000).

18. Hanahan D, Weinberg RA, Hallmarks of cancer: the next generation, Cell, 5, 646-674 (2011).

19. Reinhart BJ, et al., The 21-nucleotide let-7 RNA regulates developmental timing in Caenorhabditis elegans, Nature, 6772, 901-906 (2000).

20. Calin GA, et al., Human microRNA genes are frequently located at fragile sites and genomic regions involved in cancers, Proc. Natl. Acad. Sci. U.S.A., 9, 2999-3004 (2004).

21. Johnson SM, et al., RAS is regulated by the let-7 microRNA family, Cell, 5, 635-647 (2005).

22. Reeves R, Molecular biology of HMGA proteins: hubs of nuclear function, Gene, 1-2, 63-81 (2001).

23. Sarhadi VK, et al., Increased expression of high mobility group $A$ proteins in lung cancer, J. Pathol., 2, 206-212 (2006).

24. Faraoni I, et al., miR-155 gene: a typical multifunctional microRNA, Biochim. Biophys. Acta, 6, 497-505 (2009).

25. Vigorito E, et al., miR-155: an ancient regulator of the immune system, Immunol. Rev., 1, 146-157 (2013).

26. Higgs G, Slack $f$, The multiple roles of microRNA-155 in oncogenesis, J. Clin. Bioinforma, 1, 17 (2013).

27. Chen Y, Gao DY, Huang L, In vivo delivery of miRNAs for cancer therapy: challenges and strategies,

Adv. Drug Deliv. Rev., 81, 128-141 (2015).

28. Naidu S, Magee P, Garofalo M, MiRNA-based therapeutic intervention of cancer, J. Hematol. Oncol., 8, 68 (2015).

29. Broderick JA, Zamore PD, MicroRNA therapeutics, Gene Ther., 12, 1104-1110 (2011).

30. Bader AG, miR-34 - a microRNA replacement therapy is headed to the clinic, Front Genet., 3, 120 (2012).

31. Van Roosbroeck K, et al., Combining anti-miR-155 with chemotherapy for the treatment of lung cancers. Clin Cancer Res, doi: 10.1158/1078-0432.CCR-16-1025 (2016).

32. Xie $\mathrm{K}$, et al., A functional variant in miR-155 regulation region contributes to lung cancer risk and survival, Oncotarget, 40, 42781-42792 (2015)

33. Zheng SR, et al., Effects of miR-155 antisense oligonucleotide on breast carcinoma cell line MDA-MB-157 and implanted tumors, Asian Pac. J. Cancer Prev., 4, 2361-2366 (2013).

34. Babar IA, et al., Nanoparticle-based therapy in an in vivo microRNA-155 (miR-155)-dependent mouse model of lymphoma, Proc. Natl. Acad. Sci. U.S.A., 26, E1695-E1704 (2012).

35. Kasinski AL, et al., A combinatorial microRNA therapeutics approach to suppressing non-small cell lung cancer, Oncogene, 27, 3547-3555 (2015).

36. Cortez MA, et al., Therapeutic delivery of miR-200c enhances radiosensitivity in lung cancer, Mol. Ther., 8, 1494-1503 (2014).
37. Sicard F, et al., Targeting miR-21 for the therapy of pancreatic cancer. Mol. Ther., 5, 986-994 (2013).

38. Li Q, et al., MicroRNA-25 functions as a potential tumor suppressor in colon cancer by targeting Smad7, Cancer Lett, 1, 168-174 (2013).

39. Lee YS, et al., Depletion of human micro-RNA miR-125b reveals that it is critical for the proliferation of differentiated cells but not for the down-regulation of putative targets during differentiation, J. Biol. Chem., 17, 16635-16641 (2005).

40. Kloosterman WP, et al., Targeted inhibition of miRNA maturation with morpholinos reveals a role for $m i R-375$ in pancreatic islet development, PLoS Biol., 8, e203 (2007).

41. Gantier MP, et al., Analysis of microRNA turnover in mammalian cells following Dicer1 ablation. Nucleic Acids Res., 13, 5692-5703 (2011).

42. Hutvagner G, et al., Sequence-specific inhibition of small RNA function, PLoS Biol, 4, E98 (2004).

43. Krutzfeldt J, et al., Silencing of microRNAs in vivo with ' antagomirs', Nature, 7068, 685-689 (2005).

44. Esau C, et al., miR-122 regulation of lipid metabolism revealed by in vivo antisense targeting, Cell Metab., 2, 87-98 (2006).

45. Orom UA, Kauppinen S, Lund AH, LNA-modified oligonucleotides mediate specific inhibition of microRNA function, Gene, 372, 137-141 (2006).

46. Ebert MS, Neilson JR, Sharp PA, MicroRNA sponges: competitive inhibitors of small RNAs in mammalian cells, Nat. Methods, 9, 721-726 (2007).

47. Kluiver J, et al., Rapid generation of microRNA sponges for microRNA inhibition, PLoS One, 1, e29275 (2012).

48. Wang Z, The principles of MiRNA-masking antisense oligonucleotides technology, Methods Mol. Biol., 676, 43-49 (2011).

49. Costa PM, Pedroso de Lima MC, MicroRNAs as Molecular Targets for Cancer Therapy: On the Modulation of MicroRNA Expression, Pharmaceuticals (Basel), 10, 1195-1220 (2013).

50. Henry JC, Azevedo-Pouly AC, Schmittgen TD, MicroRNA replacement therapy for cancer, Pharm. Res., 12, 3030-3042 (2011).

51. Chorn G, et al., Single-stranded microRNA mimics, RNA, 10, 1796-1804 (2012).

52. Esau CC, Monia BP, Therapeutic potential for microRNAs, Adv. Drug Deliv. Rev., 2-3, 101-114 (2007).

53. Rossbach M, Therapeutic implications of microRNAs in human cancer, Journal of Nucleic Acids Investigation, 1, 17-22 (2011).

54. Janssen HL, et al., Treatment of HCV infection by targeting microRNA, N. Engl. J. Med., 18, 1685-1694 (2013).

55. Lieberman J, Sarnow p, Micromanaging hepatitis $C$ virus, N. Engl. J. Med., 18, 1741-1743 (2013). 